\title{
Daytime sleepiness during Ramadan intermittent fasting: polysomnographic and quantitative waking EEG study
}

\author{
RACHIDA ROKY ${ }^{1}$, FLORIAN CHAPOTOT ${ }^{2}$, MAJDA TAOUDI \\ BENCHEKROUN ${ }^{1}$, BRAHIM BENAJI ${ }^{1}$, FARID HAKKOU ${ }^{1}$, \\ HASSAN ELKHALIFI ${ }^{1}$ and ALAIN BUGUET ${ }^{2,3}$ \\ ${ }^{1}$ Department of Pharmacology, Faculty of Medicine and Pharmacy, Casablanca, Morocco, ${ }^{2}$ Département des Facteurs Humains, Centre de \\ Recherches du Service de Santé des Armées, La Tronche, France, ${ }^{3}$ Present address: Institut de Médecine Tropicale du Service de Santé des Armées, \\ Le Pharo, Marseille, France
}

Accepted in revised form 3 January 2003; received 17 April 2002

SUMMARY During the lunar month of Ramadan, Muslims abstain from eating, drinking and smoking from sunrise to sunset. We reported previously that Ramadan provokes a shortening in nocturnal total sleep time by $40 \mathrm{~min}$, an increase in sleep latency, and a decrease in slow-wave sleep (SWS) and rapid eye movement (REM) sleep duration during Ramadan. During the same study, the effects of Ramadan intermittent fasting on daytime sleepiness were also investigated in eight healthy young male subjects using a quantitative waking electroencephalograph (EEG) analysis following the multiple sleep latency test (MSLT) procedure. This procedure was combined with subjective alertness and mood ratings and was conducted during four successive experimental sessions: (1) baseline (BL) 15 days before Ramadan, (2) beginning of Ramadan (R11) on the 11th day of Ramadan, (3) end of Ramadan (R25) on the 25th day of Ramadan, (4) recovery 2 weeks after Ramadan (AR). During each session, four 20-min nap opportunities (MSLTs) were given at 10:00, 12:00, 14:00 and 16:00 $\mathrm{h}$ and were preceded by rectal temperature readings. Nocturnal sleep was recorded before each daytime session. Subjective daytime alertness did not change in R25 but decreased in R11 at 12:00 h, and subjective mood decreased at 16:00 h, both in R11 and R25. During the MSLT, mean sleep latency decreased by an average of $2 \mathrm{~min}$ in R11 (especially at 10:00 and 16:00 h) and 6 min in R25 (especially at 10:00 and 12:00 h) compared with BL. There was an increase in the daily mean of waking EEG absolute power in the theta $(5.5-8.5 \mathrm{~Hz})$ frequency band. Significant correlations were found between sleep latency during the MSLT and the waking EEG absolute power of the fast alpha (10.5$12.5 \mathrm{~Hz})$, sigma $(11.5-15.5 \mathrm{~Hz})$ and beta $(12.5-30 \mathrm{~Hz})$ frequency bands. Sleep latency was also related to rectal temperature. In conclusion, Ramadan diurnal fasting induced an increase in subjective and objective daytime sleepiness associated with changes in diurnal rectal temperature.

KEYWORDS Islamic fasting, MSLT, sleepiness, spectral analysis, visual analogue scale

Correspondence: Rachida Roky, PhD, Department of Pharmacology, Faculty of Medicine and Pharmacy, 19, rue Tarik Bnou Ziad, Casablanca, Morocco. Tel.: +212 224712 89; fax: +212 224712 90; e-mail: rroky2001@yahoo.fr

\section{INTRODUCTION}

Each year during the lunar month of Ramadan, healthy Muslims abstain from eating, drinking, smoking and sexual activity in the daytime. The changes in meal and activity schedules induce chronobiological and metabolic modifications, also related to behavioural changes. 
The delay in sleep onset has been rendered responsible for a shift towards the evening chronotype in Muslims observing Ramadan (Roky et al. 2001; Taoudi Benchekroun et al. 1999). Accordingly, several chronobiological changes have been reported, such as a decrease in the amplitude and a delay in the acrophase of the circadian oscillation of plasma melatonin, cortisol and glucose and that of body temperature (Al-Hadramy et al. 1988; Bogdan et al. 2001; Bouchareb et al. 1997; Iraki et al. 1997; Roky et al. 2000, 2001). The diurnal abstinence from meals leads also to a decrease in the diurnal resting metabolic rate (Elati et al. 1995) and diurnal plasma glucose (Iraki et al. 1997).

Behavioural changes, marked by an increased irritability (Kadri et al. 2000) and a decrease in subjective alertness (Roky et al. 2000), have been attributed to the abstinence from nicotine and coffee (Kadri et al. 2000). There is also evidence that performance and security at work are impaired. Traffic accidents increase (Shanks et al. 1994) and learning performance (Afifi 1997) and muscular force (Bigard et al. 1998; Boussif et al. 1996) are diminished.

In a previous study, our team confirmed that Ramadan fasting is accompanied by impairment in alertness, assessed by visual analogue scales (VAS), at 09:00 and 16:00 h without any change in psychomotor performance estimated from movement reaction time and critical frequency fusion tests (Roky et al. 2000). The apparent discrepancy between the result of VAS and psychomotor tests was attributed to a practising effect, which is commonly observed in the latter tests (Monk 1994). Other studies have shown that psychomotor performance, such as critical flicker fusion (Ali and Amir 1989) and memory (Hakkou et al. 1988) are impaired by Ramadan fasting. For Lagarde et al. (1996), subjective alertness decreased only at the beginning and not at the end of Ramadan, suggesting to the authors an adaptation mechanism to intermittent fasting. However, our team did not retrieve such an adaptation (Roky et al. 2000). This divergence may be due to the different methods of assessing subjective alertness.

Therefore, we used an objective measure of excessive daytime sleepiness, the multiple sleep latency test (MSLT), a test widely used to study the effects of sleep deprivation (Bonnet and Arand 1995; Roehrs et al. 2000). However, its application to mild sleep-wake cycle disturbances such as those observed during Ramadan (Roky et al. 2001) is far less documented. The MSLT consists of a daytime polysomnographic (PSG) recording, during a series of 20-min nap opportunities during which sleep latency is determined precisely. Other parameters such as wake efficiency (100 minus percentage of sleep) and sleep onset frequency (number of sleep onsets within the series of nap opportunities), were also analysed (Clodoré et al. 1990; Pollak 1997).

Sleepiness is also reflected in the waking EEG, as shown by sleep deprivation during which an increase in several EEG frequency bands occurs (delta, alpha, theta, beta) (Aeschbach et al. 1997; Lorenzo et al. 1995). Therefore, in the present study, the MSLT was combined with a quantitative analysis of the waking EEG activity.
Rectal temperature can be taken as a marker of metabolic processes, as its variations are related to metabolic changes (Aschoff and Pohl 1970). It has also been shown to change with alertness (Benoit and Foret 1988; Monk 1994). In order to demonstrate which of the alertness measures yields the closest relationship with body temperature, Spearman correlation between this variable and MSLT, subjective alertness (VAS) and waking EEG activity was calculated.

The main purpose of the present study was to measure daytime sleepiness objectively during Ramadan, using the MSLT technique, and to correlate the MSLT data with subjective data and rectal temperature.

\section{METHODS}

\section{Subjects}

Eight Muslims (20-28 years old) gave their informed written consent to participate in the protocol, which was approved by the University of Casablanca Ethics Committee and the Religious Committee of the Hassan II Foundation for Scientific and Medical Research on Ramadan. After medical and biological screening, healthy subjects were selected on their regular schedules regarding meal (breakfast at 07:00 \pm $1 \mathrm{~h}$, lunch at 12:00 $\pm 1 \mathrm{~h}$ and dinner at 20:00 $\pm 1 \mathrm{~h}$ ) and sleep (sleep between 23:00 and 07:00 $\pm 1 \mathrm{~h}$ ). They were also selected on their stable habits during Ramadan regarding meals (two nocturnal meals: break of fasting at 18:00 h; night meal between 22:00 and 24:00 h) and sleeping hours (between 24:00 \pm 1 and 08:00 $\pm 1 \mathrm{~h}$ ). The subjects were not regular nappers and belonged to the intermediate chronotype according to the scores obtained on completion of the morningness/eveningness questionnaire of Horne and Östberg (1976). They were non-smokers and were not addicted to caffeine or alcohol-containing beverages.

\section{General protocol}

Ramadan occurs in the ninth month of the lunar calendar that advances 12 days every year in relation to the Gregorian calendar. This study was performed between 19 December 1997, and the 15 February 1998, Ramadan taking place between 31 December 1997 and 29 January 1998.

The subjects underwent four continuous 24-h PSG recording sessions: (1) baseline 12 days before Ramadan (BL), (2) beginning of Ramadan on the 11th day of Ramadan (R11), (3) end of Ramadan on the 25th day of Ramadan (R25), (4) and 2 weeks after Ramadan (AR). Each experimental recording session started with a nocturnal sleep scheduled between 23:30 and 7:30 h. On the following day, subjects had four nap opportunities given at 10:00, 12:00, 14:00 and 16:00 $\mathrm{h}$ in accordance with the guidelines for the MSLT (Carskadon et al. 1986). Five minutes before the beginning of the MSLT, subjects rated their subjective alertness and mood on VAS. They were then asked to remove their shoes and lie in bed. The test began with the standard instruction to keep the eyes closes 
and not to resist sleep. Immediately after, the lights were turned off. Due to the portable PSG recording technique used, the observer being blind to the state of vigilance of the subject, the test ended $20 \mathrm{~min}$ later. Therefore, when sleep occurred during the nap opportunity, it was always shorter than $20 \mathrm{~min}$. Between the tests, subjects were continuously watched by one investigator to prevent them from sleeping or having activities other than reading, watching TV or playing card, as MSLT scores are influenced by preceding activity (Bonnet and Arand 1998). Furthermore, the absence of any sleep episode between the nap opportunities was verified afterwards by inspection of the daytime PSG recordings.

During the experimental days, daily meal composition was maintained constant in both fasting and non-fasting days and only meals schedule changed. During non-fasting days (BL, AR), meals were scheduled as follows: breakfast at 08:00 h, lunch at 12:30 h, light snack at 17:00 h and dinner at 20:30 h. During both Ramadan sessions, they neither ate nor drank during the daytime. Meals schedule was: break of fasting at 18:00 $\mathrm{h}$ at the beginning of Ramadan and 18:30 $\mathrm{h}$ at the end of Ramadan, light snack at 21:00 h, and dinner at 22:30 h. When at home, the subjects were asked to comply with the meal composition and with the schedule adopted during the laboratory investigation.

\section{Polysomnographic recordings}

The PSG recordings are described in a previous publication (Roky et al. 2001). Briefly, electroencephalogram (EEG), electro-oculogram (EOG) and electromyogram (EMG) were recorded on eight-channel ambulatory Oxford Medilog MR$9000 \mathrm{II}^{\circledR}$ recorders (Oxford Instruments, Abington, UK). The EEG electrodes were placed at sites $\mathrm{C} 3, \mathrm{C}_{4}, \mathrm{~A}_{1}, \mathrm{~A}_{2}, \mathrm{Cz}$ and $\mathrm{Fz}$. The $\mathrm{C}_{3}$ and $\mathrm{C}_{4}$ electrodes were connected to the linked ears reference $\mathrm{A} 1$ and $\mathrm{A}_{2}$. The analogue signals were stored on C-120 tapes which were then converted to digital with an eightbit resolution and a sampling frequency of $128 \mathrm{~Hz}$ using the Vision ${ }^{\circledR}$ software (Oxford Instruments).

\section{Sleep-stage scoring and quantitative waking EEG analysis}

Sleep stage scoring and quantitative analyses of the serial waking EEG were performed with the ERA-Profiler ${ }^{\odot}$ 2.1 software package (PhiTools ${ }^{\circledR}$, Strasbourg, France). Nocturnal and MSLT PSG recordings were visually scored in 20-s epochs following classical criteria for wakefulness, stages 1, 2, 3 and 4 of non-rapid eye movement sleep (NREM sleep, stages 3 and 4 constituting SWS), and REM sleep (Rechtschaffen and Kales 1968). Sleep onset was defined by the beginning of three consecutive 20-s epochs of stage 1 or at least one epoch of any other deeper sleep stage. Artifacts (eye blinks, movements and electrode detachments) were detected automatically through combining digital filtering and background-dependent amplitude thresholds and were subsequently inspected by an expert. The 2-s EEG epochs overlapping with artifacts did not exceed $25 \%$ of the recording sequences and were discarded from further analysis. Absolute EEG power $\left(\mu \mathrm{V}^{2}\right)$ spectra of the $\mathrm{C}_{3}-$ $\mathrm{A}_{1-2}$ and $\mathrm{C}_{4}-\mathrm{A}_{1-2}$ derivations were computed between 0.5 and $30 \mathrm{~Hz}$ on the remaining 2-s epochs using a fast Fourier transform algorithm. The 2-s spectra belonging to the waking phase (20-s epochs scored as wake) of each MSLT were subsequently averaged, yielding in a mean estimate of the waking EEG activity during each test. Absolute power of the delta $(0.5-5.5 \mathrm{~Hz})$, theta $(5.5-8.5 \mathrm{~Hz})$, alpha $(8.5-12.5 \mathrm{~Hz})$, sigma $(11.5-15.5 \mathrm{~Hz})$ and beta $(12.5-30 \mathrm{~Hz})$ frequency bands was computed by summing up powers in the $0.5-\mathrm{Hz}$ frequency bins belonging to the corresponding frequency band, the upper bin being excluded. Frequency band limits were adapted for the study of waking EEG (Chapotot et al. 2001) and additionally included the classical sigma $(11.5-15.5 \mathrm{~Hz})$ band for sleep studies.

\section{Subjective mood and alertness}

Global subjective alertness and mood were calculated as shown by Monk (1989). Eight univocal VAS ratings were used. Four of them concerned subjective alertness (alertness, tiredness, sleepiness and awkwardness) and four subjective mood and affect (happiness, sadness, calmness and tension).

\section{Core temperature measurements}

As previously described (Roky et al. 2001), rectal temperature was continuously recorded with a thermistor probe, which was inserted $12 \mathrm{~cm}$ into the rectum and attached to a light portable belt recorder, and was noted before each nap during the four experimental sessions.

\section{Statistical analysis}

The influence of Ramadan on MSLT scores, quantitative waking EEG measurements and VAS scores were analysed using a non-parametric Friedman analysis of variance (ANOVA), with the experimental sessions as factors. When the ANOvA indicated significant differences, post hoc multiple comparisons between sessions for the four naps and for each nap of a given session were performed using Wilcoxon paired $t$-tests.

Two-way Anova was also used with the time of day and Ramadan session as factors.

Statistical comparison of waking EEG absolute powers was performed after a logarithmic transformation ensuring a normal residual distribution. Non-parametric Spearman correlation coefficient was used to determine the relationships between MSLT scores and rectal temperature, and between diurnal waking EEG activity and nocturnal sleep.

\section{RESULTS}

\section{Nocturnal sleep}

The effect of Ramadan on nocturnal sleep was detailed in a previous paper (Roky et al. 2001). Briefly, total sleep time 
decreased (418 \pm 12 versus $362 \pm 26 \mathrm{~min}$ ) and sleep latency increased $(27.7 \pm 9$ versus $85.1 \pm 29 \mathrm{~min})$ at the end of Ramadan. The proportion of NREM sleep increased during Ramadan and its structure changed, with an increased duration in stage 2 and a decrease in SWS. The REM sleep duration and proportion decreased during Ramadan.

\section{Daytime sleepiness, mood, diurnal waking EEG activity and rectal temperature MSLT scores}

Sleep onset frequency (Fig. 1) was not changed during Ramadan in comparison with BL, but was higher in R25 compared with $\operatorname{AR}(t=75, P=0.004)$. Sleep latency (Fig. 1) varied between sessions $\left(\chi^{2}=12.3, P=0.007\right)$, with a decrease at the end of Ramadan (R25; $11 \mathrm{~min} \pm 2.5 \mathrm{~min}$ ) in comparison with baseline (17 $\mathrm{min} \pm 2 \mathrm{~min} ; t=3.9, P=0.0009)$. It did not change at the beginning of Ramadan (R11) and resumed BL values in AR. Time-of-day effects were observed in the nonfasting condition (BL and AR), sleep latency being shorter at $14: 00 \mathrm{~h}$ than at $10: 00 \mathrm{~h}(t=0, P=0.027$ in $\mathrm{BL} ; t=0$, $P=0.018$ in AR $)$ and $16: 00 \mathrm{~h}(t=2, P=0.042$ in $\mathrm{BL}$ and $t=0, P=0.042$ in AR). During Ramadan, the decrease in sleep latency was more important at $10: 00 \mathrm{~h} \quad(t=3.04$, $P=0.018)$ and at $12: 00 \mathrm{~h}(t=5.2, P=0.0012)$.

Wake duration (Fig. 1) decreased $\left(\chi^{2}=12.6, P=0.006\right)$ during Ramadan, especially in R25 (83\% in BL, 75\% in R11, $61 \%$ in R25 and $78 \%$ in AR). Time-of-day effect was significant for all sessions. In non-fasting conditions (BL and AR), wake duration dipped at 14:00 h, but at 12:00 h at the end of Ramadan (R25). In Ramadan condition, the decrease in wake duration was larger at 10:00 h $(t=0, P=0.011)$ and $12: 00 \mathrm{~h}(t=3, P=0.02)$ than at $16: 00 \mathrm{~h}(F=2.2, P=0.03)$. In $\mathrm{AR}$, wake duration resumed $\mathrm{BL}$ values.

The duration of stage 1 (Fig. 1) did not change from BL to Ramadan, but it was longer in R11 and R25 in comparison with $\operatorname{AR}(t=66, P=0.028 ; t=69, P=0.02$; mean stage 1 duration was: $6 \pm 3.2 \%$ in BL, $11 \pm 4 \%$ in R $11,18 \pm 2.5 \%$ in R25 and $6 \pm 2.1 \%$ in AR). Slow-wave sleep and REMsleep did not occur in any of the naps.

\section{Subjective measure of alertness and mood}

In non-fasting conditions, subjective alertness followed the variations seen with the MSLT measures (Fig. 2). In BL and AR, subjective alertness was lower at 14:00 h $(t=1, P=0.02$; $t=5, P=0.038)$ than at $12: 00 \mathrm{~h}$. This was not true during Ramadan, as subjective alertness decreased only at 12:00 $\mathrm{h}$ in R11 $(t=4, P=0.049)$ and increased at $14: 00 \mathrm{~h}(t=2.5$, $P=0.029)$ in R25.

Subjective mood decreased slightly at 16:00 h in R11 and $\mathrm{R} 25$. It was greater at 12:00 $\mathrm{h}$ in Ramadan than in $\mathrm{AR}(t=2$, $P=0.025$ in $\mathrm{R} 11$ and $t=6, P=0.05$ in R25). After Ramadan, mood resumed BL values at 10:00, 14:00 and 16:00 h but was low at 12:00 h $(t=2, P=0.025)$.

Subjective alertness and mood were positively correlated. Both variables were also positively correlated to the MSLT measure of sleep latency (Table 1).

\section{Diurnal waking EEG activity}

During the waking epochs of the daytime naps, the EEG absolute powers in the delta $(0.5-5.5 \mathrm{~Hz})$, alpha $(8.5-12.5 \mathrm{~Hz})$, sigma $(11.5-15.5 \mathrm{~Hz})$, beta $(12.5-30 \mathrm{~Hz})$ frequency bands, as well as the overall absolute power $(0.5-30 \mathrm{~Hz})$, were not affected by Ramadan. In contrast, the waking EEG activity in the theta $(5.5-8.5 \mathrm{~Hz})$ frequency band increased during Ramadan fasting as compared with $\mathrm{BL}(t=3.24, P=0.006)$.

In addition, positive correlations were found between the MSLT measures of sleep latency and the waking EEG absolute power in the fast alpha $(10.5-12.5 \mathrm{~Hz})(r=0.25, P=0.02)$,
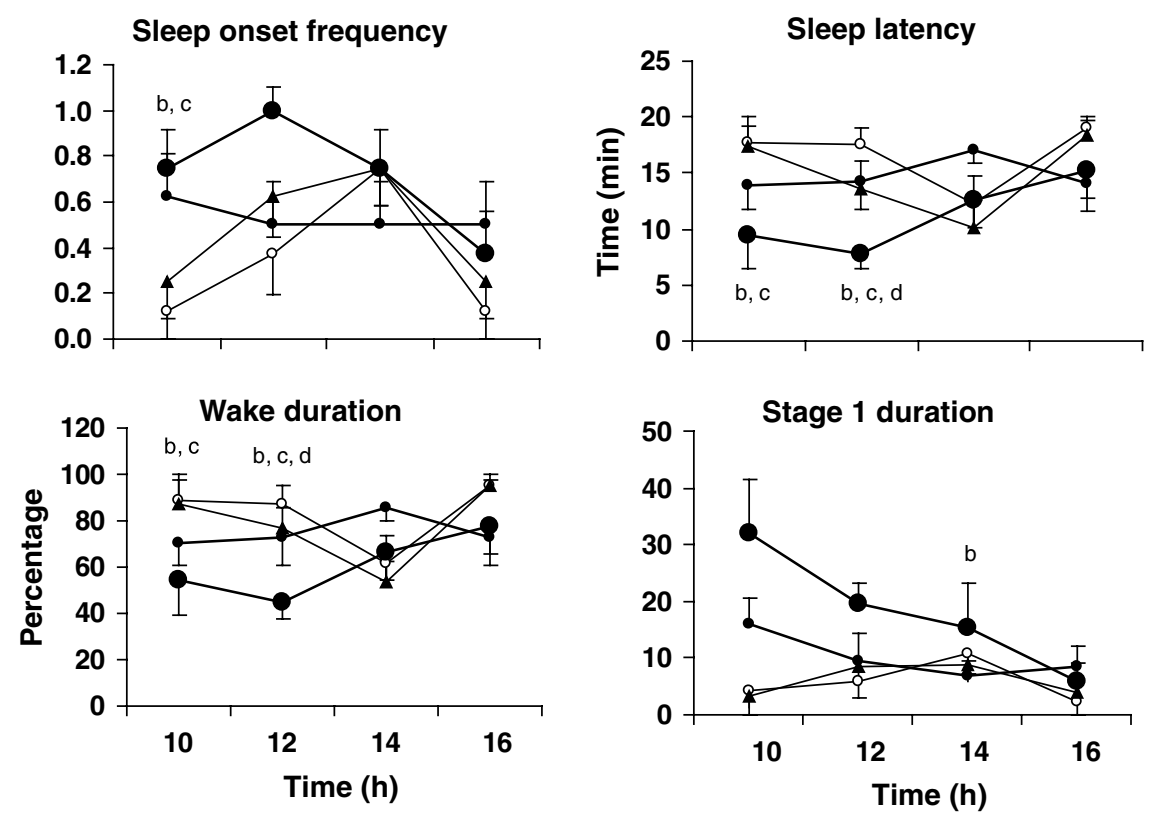

Figure 1. Mean and standard error of the mean of the multiple sleep latency text scores (sleep onset frequency, sleep latency, proportion of wakefulness and stage 1) in baseline (BL, open circles), at the beginning (R11, small solid circles) and the end (R25, large solid circles) of Ramadan, and after Ramadan (AR, solid triangles). a, b, c, d, indicate significant effects $(P<0.05)$ for the post hoc analysis (a, BL versus R11; b, BL versus R25; c, AR versus R25; d, R11 versus R25). 
Figure 2. Mean and standard error the mean of subjective alertness and mood values assessed by the visual analogue scale before naps in baseline (BL, open circles), at the beginning (R11, small solid circles), and the end (R25, large solid circles) of Ramadan and after Ramadan (AR, solid triangles). The unit of the $y$-axis is arbitrary. a, b, c, d, indicate significant effects $(P<0.05)$ for the post hoc analysis (a, BL versus R11; b, BL versus R25; c, $A R$ versus R11; d, AR versus R25; f, $\mathrm{BL} / \mathrm{AR}$ ).
Subjective alertness

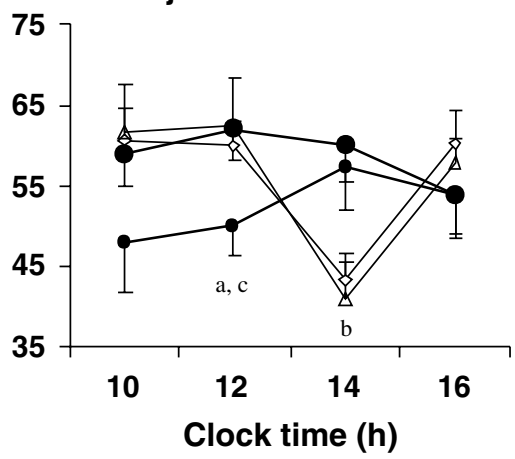

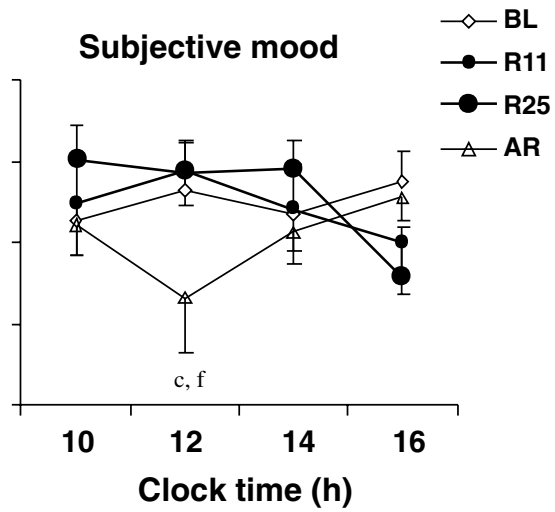

Table 1 Spearman correlations between sleep latency and the subjective alertness and mood, the power density of the waking electroencephalograph. Only significant correlations are reported

\begin{tabular}{llll}
\hline & Rho & Z-score & $P$-value \\
\hline Sleep latency versus & & & \\
$\quad$ Subjective alertness & 0.23 & 2.6 & 0.009 \\
Subjective mood & 0.18 & 2.1 & 0.038 \\
Alpha 2 (10.5-12.5 Hz) & 0.25 & 2.2 & 0.02 \\
Beta (12.5-30 Hz) & 0.27 & 2.3 & 0.02 \\
$\quad$ Sigma (11.5-15.5 Hz) & 0.29 & 2.6 & 0.01 \\
$\quad$ Subjective alertness versus & & & \\
$\quad$ Subjective mood & 0.28 & 3.1 & 0.002 \\
\hline
\end{tabular}

beta $(r=0.27, P=0.02)$ and sigma $(r=0.29, P=0.01)$ frequency bands (Table 1).

\section{Rectal temperature and correlations with sleepiness measures}

Rectal temperature decreased during Ramadan (R11: $t=68$, $P=0.015$; R25: $t=94, P=0.001)$ (Fig. 3, Table 2), especially at $16: 00 \mathrm{~h}$ in R25 ( $t=0, P=0.012)$ compared with BL. Rectal temperature correlated best with sleep latency $(r=0.35, P=0.0002)$. Higher rectal temperature corresponded to a larger delay in sleep onset and to a longer wake duration prior to sleep during the nap episodes (Table 2). Conversely, greater rectal temperature values correlated with shorter stage 2 duration during nap sleep.

Although the subjective measures of alertness and mood did not correlate with rectal temperature (Table 2), a positive correlation between rectal temperature and sigma (11.5$15.5 \mathrm{~Hz}$ ) power was observed.

\section{DISCUSSION}

This study showed an increase in daytime sleepiness, especially at 10:00 and 12:00 h at the end of Ramadan, and a decrease in daytime body temperature during Ramadan. These results are in agreement with reports of a decrease in psychomotor, learning and muscular performances during Ramadan (Afifi 1997; Bigard et al. 1998; Boussif et al. 1996; Roky et al. 2000). This increase in sleepiness, especially in the morning, could be due to the

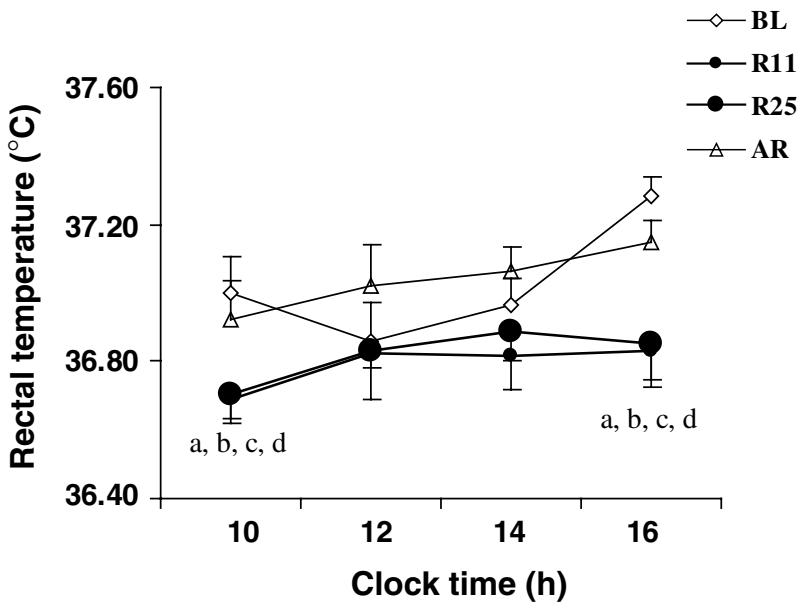

Figure 3. Mean and standard error of the mean of rectal temperature before naps in baseline (BL, open circles), at the beginning (R11, small solid circles), the end (R25, large solid circles) of Ramadan and after Ramadan (AR, solid triangles). a, b, c, d, indicate significant effects $(P<0.05)$ for the post hoc analysis (a, BL versus R11; b, BL versus R25; c, AR versus R11; d, AR versus R25).

Table 2 Spearman correlations between rectal temperature and multiple sleep latency test data, subjective alertness and mood and waking electroencephalograph bands

\begin{tabular}{lccl}
\hline & Rho & Z-score & $P$-value \\
\hline Rectal temperature versus & & & \\
Sleep latency & 0.35 & 3.7 & 0.0002 \\
Sleep onset & -0.2 & -2.1 & 0.032 \\
Stage 1 duration & -0.16 & -1.7 & $\mathrm{NS}$ \\
Stage 2 duration & -0.19 & -2 & 0.04 \\
Wake duration & 0.34 & 3.5 & 0.0004 \\
Subjective alertness & 0.056 & 0.58 & $\mathrm{NS}$ \\
Subjective mood & 0.09 & 0.89 & $\mathrm{NS}$ \\
Beta 1 (12.5-15 Hz) & 0.24 & 2.0 & 0.045 \\
Sigma (11.5-15.5 Hz) & 0.25 & 2.2 & 0.03 \\
\hline
\end{tabular}

decrease of the nocturnal sleep duration, the delay in rising time, and the absence of breakfast and caffeine intake.

The MSLT measures of sleep latency and the duration of wake and stage 1 during naps varied in the same way during Ramadan. Although wake efficiency has been thought to 
represent the best parameter to appreciate daytime sleepiness from MSLT data (Pollak 1997), the percentage of wake and sleep latency gave a similar estimate of sleepiness. Also, we could not confirm that the number of sleep onsets during the naps was a better measure in evaluating sleepiness, as had been suggested by Clodoré et al. (1990).

Technically, the use of the portable polygraph equipment made necessary to end the MSLT after $20 \mathrm{~min}$. Therefore, the subjects could only sleep less than $20 \mathrm{~min}$. As in the usual MSLT procedure, the amount of sleep in one nap could have influenced that of the following nap. However, the decrease in sleep latency in non-fasting conditions at 14:00 h, which represented the third nap, demonstrated that this was not the case, in accordance with Pollak (1997).

Subjective assessments showed an increase in sleepiness at the beginning of Ramadan, the MSLT measures at the end. However, the measures from both techniques were correlated, especially in non-fasting conditions with an increase in sleepiness at 14:00 $\mathrm{h}$. The results of the subjective evaluation suggest that there was an adaptation to Ramadan fasting as was reported earlier by Lagarde et al. (1996). The result of the MSLT did not confirm this observation.

Several studies have demonstrated that MSLT and subjective measures of sleepiness do not always correlate, especially in young people (Manni et al. 1991), even after sleep deprivation (Devoto et al. 1999). Subjective evaluations are more influenced by psychological factors than is the MSLT (Olson et al. 1998). Effectively, the feeling of pride and satisfaction that accompanies the religious fasting during Ramadan may interfere with subjective ratings of alertness, although mood did not change during Ramadan. Subjective measures of sleepiness and MSLT may also evaluate different processes, the former measuring subjective feeling of fatigue, the latter the tendency to sleep.

Another major finding was the slowing in daytime cerebral activity during Ramadan, marked by an increase in the EEG theta band power density during the wakefulness episodes of the naps. This finding confirms earlier report of increased theta activity during a situation of increased sleepiness (Aeschbach et al. 1999; Cajochen et al. 1995; Corsi-Cabrera et al. 1994; Torsvall and Akerstedt 1988). In these studies, both the theta and alpha bands increased, especially when subjects were examined in active wakefulness with the eyes opened. In other recent studies, only the theta band activity was shown to increase when alertness decreased (Aeschbach et al. 2001; Cajochen et al. 1999; Lafrance and Dumont 2000), even in case of moderate sleepiness. There are controversies in the literature as to the modifications of the EEG alpha band in conditions inducing sleepiness. Cajochen et al. (1995) believed that the increase in the range of $6.25-9.0 \mathrm{~Hz}$ reported after sleep deprivation relates more to the theta frequency than the alpha frequency band. In our protocol, however, the positive correlation between the fast alpha activity $(10.5-12.5 \mathrm{~Hz})$ and sleep latency during Ramadan demonstrated that this activity decreases when sleepiness increases, at least when subjects are recorded with the eyes closed. The waking alpha frequency has also been found to decrease after the induction of sleepiness by L-tryptophan administration (Spinweber et al. 1983) and after sleep deprivation (Brunner et al. 1993; Lorenzo et al. 1995). However, a lack of correlation between the alpha band power and neurobehavioural performance has also been reported (Cajochen et al. 1999). Taken together, these findings suggest that the theta activity is better than that of the alpha band in evaluating the level of sleepiness, because it always increases with sleepiness independently of the recording situation.

Aeschbach et al. (1999) hypothesized that theta activity is correlated to objectively measurable sleep propensity and that high-frequency alpha activity is correlated to the perception of the arousal level. In the present study, however, theta power was not significantly correlated to sleep latency.

Diurnal rectal temperature was decreased during Ramadan in relation to the decrease in the resting metabolic rate and the diurnal the energy expenditure (Elati et al. 1995; Sweileh et al. 1992). Rectal temperature was correlated to sleep latency, alertness, mood, sigma and beta activities, suggesting that the increase in sleepiness is related to metabolic changes. Effectively, metabolic changes influence cerebral activity: increased theta activity is associated with hypometabolism in the medial thalamus (Paus et al. 1997) and with hypoglycaemia (Bjorgaas et al. 1998; Cox et al. 2000).

In conclusion, sleepiness estimated with objective and subjective techniques increased during Ramadan. This increase was related to the metabolic changes observed during the intermittent fasting.

\section{ACKNOWLEDGEMENTS}

This work was supported by the Hassan II Foundation for Scientific and Medical Research on Ramadan (Casablanca, Morocco) and the Centre de recherches du service de santé des armées Emile Pardé (La Tronche, France). The authors are indebted to the volunteers and their families who supported the experimental design for more than 2 months. They are also grateful for the skilled assistance of Nadine Fidier and Alain Roux, and to Prof. Benchekroun, who is admired for his interest in the relations between Religion and Science.

\section{REFERENCES}

Aeschbach, D., Matthews, J. R., Postolache, T. T., Jackson, M. A., Giesen, H. A. and Wehr, T. A. Dynamics of the human EEG during prolonged wakefulness: evidence for frequency-specific circadian and homeostatic influences. Neurosci. Lett., 1997, 239: 121-124.

Aeschbach, D., Matthews, J. R., Postolache, T. T., Jackson, M. A., Giesen, H. A. and Wehr, T. A. Two circadian rhythms in the human electroencephalogram during wakefulness. Am. J. Physiol., 1999, 277: R1771-R1779.

Aeschbach, D., Postolache, T. T., Sher, L., Matthews, J. R., Jackson, M. A. and Wehr, T. A. Evidence from the waking electroencephalogram that short sleepers live under higher homeostatic sleep pressure than long sleepers. Neuroscience, 2001, 102: 493-502.

Afifi, Z. E. M. Daily practices, study performance and health during the Ramadan fast. J. Roy. Soc. Health, 1997, 117: 231-235. 
Al-Hadramy, M. S., Zawawi, T. H. and Abdelwahab, S. M. Altered cortisol levels in relation to Ramadan. Europ. J. Clin. Nutr., 1988, 42: 359-362.

Ali, M. R. and Amir, T. Effects of fasting on visual flicker fusion. Percept. Mot. Skills., 1989, 69: 627-631.

Aschoff, J. and Pohl, H. Rhythmic variations in energy metabolism. Fed. Proc., 1970, 29: 1541-1552.

Benoit, O. and Foret, J. Regulation circadienne des états de veille et de sommeil. Neurophysiol. Clin., 1988, 18: 403-431.

Bigard, A. X., Boussif, M., Chalabi, H. and Guézennec, C. Y. Alterations in muscular performance and orthostatic tolerance during Ramdan. Aviat. Space Environ. Med., 1998, 69: 341-346.

Bjorgaas, M., Sand, T., Vik, T. and Jorde, R. Quantitative EEG during controlled hypoglycaemia in diabetic and non-diabetic children. Diabetes Med., 1998, 15: 30-37.

Bogdan, A., Bouchareb, B. and Touitou, Y. Ramadan fasting alters endocrine and neuroendocrine circadian patterns. Meal-time as a synchronizer in humans? Life Sci., 2001, 68: 1607-1615.

Bonnet, M. H. and Arand, D. L. We are chronically sleep deprived. Sleep, 1995, 18: 908-911.

Bonnet, M. H. and Arand, D. L. Sleepiness as measured by modified multiple sleep latency testing varies as a function of preceding activity. Sleep, 1998, 21: 477-483.

Bouchareb, B., Bogdan, A. and Touitou, Y. The modifications of the hormonal temporal structure during Ramadan fasting. Chronobiol. Int., 1997, 14 (Suppl. 1): 21.

Boussif, M., Bigard, A. X., Chalabi, H. and Guézennec, C. Y. Effets du Ramadan sur les performances physiques de pilotes d'avions de combat. Méd. Aéronaut. Spatiale, 1996, 5: 166-174.

Brunner, D. P., Dijk, D. J. and Borbely, A. A. Repeated partial sleep deprivation progressively changes the EEG during sleep and wakefulness. Sleep, 1993, 16: 100-113.

Cajochen, C., Brunner, D. P., Krauchi, K., Graw, P. and Wirz-Justice, A. Power density in theta/alpha frequencies of the waking EEG progressively increases during sustained wakefulness. Sleep, 1995, 18: $890-894$

Cajochen, C., Khalsa, S. B., Wyatt, J. K., Czeisler, C. A. and Dijk, D. J. EEG and ocular correlates of circadian melatonin phase and human performance decrements during sleep loss. Am. J. Physiol., 1999, 277: R640-R649.

Carskadon, M. A., Dement, W. C., Mitler, M. M., Roth, T., Westbrook, P. R. and Keenan, S. Guidelines for the multiple sleep latency test (MSLT): a standard measure of sleepiness. Sleep, 1986, 9: $519-524$.

Chapotot, F., Buguet, A., Gronfier, C. and Brandenberger, G. Hypothalamo-pituitary-adrenal axis activity is related to the level of central arousal: effect of sleep deprivation on the association of high-frequency waking electroencephalogram with cortisol release. Neuroendocrinology, 2001, 73: 312-321.

Clodoré, M., Benoit, O., Foret, J. and Bouard, G. The multiple sleep latency test. individual variability and time of day effect in normal young adults. Sleep, 1990, 13: 385-394.

Corsi-Cabrera, M., Ponce-De-Leon, M., Juarez, J. and Ramos, J. Effects of paradoxical sleep deprivation and stress on the waking EEG of the rat. Physiol. Behav., 1994, 55: 1021-1027.

Cox, D. J., Gonder-Frederick, L. A., Kovatchev, B. P., Julian, D. M. and Clarke, W. L. Progressive hypoglycemia's impact on driving simulation performance. Occurrence, awareness and correction. Diabetes Care, 2000, 23: 163-170.

Devoto, A., Lucidi, F., Violani, C. and Bertini, M. Effects of different sleep reductions on daytime sleepiness. Sleep, 1999, 22: 336-343.

Elati, J., Beji, C. and Danguir, J. Increased fat oxidation during Ramadan fasting in healthy women: an adaptive mechanism for body weight maintenance. Am. J. Clin. Nutr., 1995, 62: 302307.

Hakkou, F., Wast, D. and Jaouen, C. Does Ramadan impair vigilance and memory. Psychopharmacol., 1988, 96: 213.
Horne, J. A. and Östberg, O. A self assessment questionnaire to determine morningness-eveningness in human circadian rhythms. Int. J. Chronobiol., 1976, 4: 97-110.

Iraki, L., Bogdan, A., Hakkou, F., Amrani, N., Abkari, A. and Touitou, Y. Ramadan diet restriction modifies the circadian time structure in humans. Study on plasma gastrin, insulin, glucose and calcium and on gastric pH. J. Clin. Endocrinol. Metab., 1997, 82: $1261-1273$.

Kadri, N., Tilane, A., El Batal, M., Taltit, Y., Tahiri, S. M. and Moussaoui, D. Irritability during the month of Ramadan. Psychosom. Med., 2000, 62: 280-285.

Lafrance, C. and Dumont, M. Diurnal variations in the waking EEG. comparisons with sleep latencies and subjective alertness. J. Sleep Res., 2000, 9: 243-248.

Lagarde, D., Batejat, D., Boussif, M., Pradella, S., Girault, S. and Huppe, M. Ramadan et vigilance. Méd. Aéronaut. Spatiale., 1996, 35: $175-182$.

Lorenzo, I., Ramos, J., Arce, C., Guevara, M. A. and Corsi-Cabrera, M. Effect of total sleep deprivation on reaction time and waking EEG activity in man. Sleep, 1995, 18: 346-354.

Manni, R., Ratti, M. T., Barzaghi, N., Galimberti, C. A., Zucca, C., Perucca, E. and Tartara, A. Daytime sleepiness in healthy University students: a multiparametric study. Ital. J. Neurol. Sci., 1991, 2: 303-309.

Monk, T. H. A visual analogue scale technique to measure global vigor and affect. Psychiatry Res., 1989, 27: 89-99.

Monk, T. H. Circadian rhythms in subjective activation, mood and performance efficiency. In: M. H. Kryger, T. Roth and W. C. Dement (Eds) Principles and Practice of Sleep Medicine. W.B. Saunders Company, Philadelphia, 1994: 321-330.

Olson, L. G., Cole, M. F. and Ambrogetti, A. Correlations among Epworth Sleepiness Scale scores, multiple sleep latency tests and psychological symptoms. J. Sleep. Res., 1998, 7: 248-253.

Paus, T., Zatorre, R. J., Hofle, N., Caramanos, Z., Gotman, J., Petrides, M. and Evens, A. C. Time related changes in neural systems underlying attention and arousal during the performance of an auditory vigilance task. J. Cogn. Neurosci., 1997, 9: 392-408.

Pollak, C. P. How should the multiple sleep latency test be analyzed? Sleep, 1997, 20: 34-39.

Rechtschaffen, A. and Kales, A. A Manual of Standardized Terminology, Techniques and Scoring System for Sleep Stages of Human Subjects. NIH Publications No. 204. Public Health Service, U.S. Government Printing Office, Washington, DC, 1968.

Roehrs, T., Turner, L. and Roth, T. Effects of sleep loss on waking actigraphy. Sleep, 2000, 23: 793-797.

Roky, R., Chapotot, F., Hakkou, F., Taoudi Benchekroun, M. and Buguet, A. Sleep during Ramadan intermittent fasting. J. Sleep Res., 2001, 10: 319-327.

Roky, R., Iraki, L., HajKhlifa, R., Lakhdar Ghazal, N. and Hakkou, F. Daytime alertness, mood, psychomotor performances, and oral temperature during Ramadan intermittent fasting. Ann. Nutr. Metab., 2000, 44: 101-107.

Shanks, N. J., Ansari, M. and Al-Kalai, D. Road traffic accident in Saudi Arabia. Public Health, 1994, 108: 27-34.

Spinweber, C. L., Ursin, R., Hilbert, R. P. and Hilderbrand, R. L. L-tryptophan: effects on daytime sleep latency and the waking EEG. Electroencephalogr. Clin. Neurophysiol., 1983, 55: 652-661.

Sweileh, N., Schnitzler, A., Hunter, G. and Davis, B. Body composition and energy metabolism in resting and exercising Muslims during Ramadan fast. J. Sports Med. Phys. Fitness, 1992, 32: 156163.

Taoudi Benchekroun, M., Roky, R., Toufik, J., Benaji, B. and Hakkou, F. Epidemiological study: chronotype and sleepiness before and during Ramadan. Thérapie, 1999, 54: 567-572.

Torsvall, L. and Akerstedt, T. Extreme sleepiness. quantification of EOG and spectral EEG parameters. Int. J. Neurosci., 1988, 38: 435 441. 